Article

\title{
Recent Advances and Applications of External Cavity-QCLs towards Hyperspectral Imaging for Standoff Detection and Real-Time Spectroscopic Sensing of Chemicals
}

\author{
Ralf Ostendorf ${ }^{1, *}$, Lorenz Butschek ${ }^{1}$, Stefan Hugger ${ }^{1}$, Frank Fuchs ${ }^{1}$, Quankui Yang ${ }^{1}$, \\ Jan Jarvis ${ }^{1}$, Christian Schilling ${ }^{1}$, Marcel Rattunde ${ }^{1}$, André Merten ${ }^{2}$, Jan Grahmann ${ }^{2}$, \\ Dusan Boskovic $^{3}$, Thorsten Tybussek ${ }^{4}$, Klaus Rieblinger ${ }^{4}$ and Joachim Wagner ${ }^{1}$ \\ 1 Fraunhofer Institute for Applied Solid State Physics IAF, Tullastrasse 72, 79108 Freiburg, Germany; \\ lorenz.butschek@iaf.fraunhofer.de (L.B.); stefan.hugger@iaf.fraunhofer.de (S.H.); \\ frank.fuchs@iaf.fraunhofer.de (F.F.); quankui.yang@iaf.fraunhofer.de (Q.Y.); \\ jan-philip.jarvis@iaf.fraunhofer.de (J.J.); Christian.schilling@iaf.fraunhofer.de (C.S.); \\ Marcel.Rattunde@iaf.fraunhofer.de (M.R.); joachim.wagner@iaf.fraunhofer.de (J.W.) \\ 2 Fraunhofer Institute for Photonic Microsystems IPMS, Maria-Reiche-Strasse 2, 01109 Dresden, Germany; \\ andre.merten@ipms.fraunhofer.de (A.M.); jan.grahmann@ipms.fraunhofer.de (J.G.) \\ 3 Fraunhofer Institute for Chemical Technology ICT, Joseph-von-Fraunhofer Str. 7, 76327 Pfinztal, Germany; \\ dusan.boskovic@ict.fraunhofer.de \\ 4 Fraunhofer Institute for Process Engineering and Packaging IVV, Giggenhauser Straße 35, 85354 Freising, \\ Germany; Thorsten.Tybussek@ivv.fraunhofer.de (T.T.); klaus.rieblinger@ivv.fraunhofer.de (K.R.) \\ * Correspondence: ralf.ostendorf@iaf.fraunhofer.de; Tel.: +49-761-5159-638
}

Received: 31 March 2016; Accepted: 30 April 2016; Published: 13 May 2016

\begin{abstract}
External-cavity quantum cascade lasers (EC-QCL) are now established as versatile wavelength-tunable light sources for analytical spectroscopy in the mid-infrared (MIR) spectral range. We report on the realization of rapid broadband spectral tuning with $\mathrm{kHz}$ scan rates by combining a QCL chip with a broad gain spectrum and a resonantly driven micro-opto-electro-mechanical (MOEMS) scanner with an integrated diffraction grating in Littrow configuration. The capability for real-time spectroscopic sensing based on MOEMS EC-QCLs is demonstrated by transmission measurements performed on polystyrene reference absorber sheets, as well as on hazardous substances, such as explosives. Furthermore, different applications for the EC-QCL technology in spectroscopic sensing are presented. These include the fields of process analysis with on- or even inline capability and imaging backscattering spectroscopy for contactless identification of solid and liquid contaminations on surfaces. Recent progress in trace detection of explosives and related precursors in relevant environments as well as advances in food quality monitoring by discriminating fresh and mold contaminated peanuts based on their MIR backscattering spectrum is shown.
\end{abstract}

Keywords: quantum cascade lasers; external cavity quantum cascade lasers; MOEMS grating; quantum cascade laser based spectroscopy; imaging laser backscattering spectroscopy; inline spectroscopic analysis

\section{Introduction}

In this paper we report on recent advances in external-cavity quantum cascade laser (EC-QCL) technology at our institute [1,2], focusing on real-time applications for broadband mid-infrared (MIR) spectroscopy. The MIR region is ideally suited for quantum cascade lasers (QCLs), covering a wavelength range from $3.3 \mu \mathrm{m}$ to around $12 \mu \mathrm{m}$, using just a single semiconductor material combination, namely GaInAs/AlInAs-on-InP [3-5]. EC-QCLs covering the MIR spectral range have 
been shown to be versatile wavelength-tunable light sources for analytical spectroscopy [6-9]. Using a multiple-stage hetero-cascading active region design, the spectral gain width of a QCL chip can be engineered to yield widely tunable EC-QCLs with a maximum wavelength coverage from, e.g., $7.6 \mu \mathrm{m}$ $\left(1315 \mathrm{~cm}^{-1}\right)$ to $11.4 \mu \mathrm{m}\left(772 \mathrm{~cm}^{-1}\right)$ [10]. This equals a relative wavelength coverage of $\Delta \lambda / \lambda \approx 40 \%$. An even wider wavelength span of $1000 \mathrm{~cm}^{-1}$ can be covered when several EC-QCL equipped with QCL chips of different wavelength coverage are combined into one system [11,12].

Optical spectroscopy in the MIR is a valuable tool in analytical chemistry, as the fundamental molecular rotational-vibrational transitions with large oscillator strength fall into this energy range. The highly characteristic absorption patterns, especially in the so-called "fingerprint region" $\left(\sim 500 \mathrm{~cm}^{-1}-1500 \mathrm{~cm}^{-1}\right)$, are well suited for identification and quantification of analytes, including the detection of small quantities of target substances within an unknown matrix. FTIR spectrometers with broadband thermal radiation sources have therefore long become an indispensable laboratory tool.

Widely tunable lasers, such as EC-QCL, offer new possibilities to MIR spectroscopy. Key advantages are, e.g., high spectral resolution, very high spectral power density and the emission of a well-defined output beam that can easily be collimated and sent over long distances or coupled efficiently into subsequent optical systems including optical fibers and waveguides. Examples of the use of EC-QCLs in analytical spectroscopy include the detection of contaminations in water supplies [13], inline-monitoring of chemical reactions [14], as well as MIR laser backscattering spectroscopy for the detection of residues of explosives on surfaces [15-18] or assessing food quality [2]. The latter three application cases will be presented and discussed in more detail in this paper.

Apart from the width of the spectral tuning range, the tuning speed of an EC-QCL can be an important factor for many applications, such as real-time monitoring and in-line process analysis. Commercially available EC-QCL systems provide wavelength tuning speeds in the range from $>0.1 \mathrm{~cm}^{-1} / \mathrm{ms}$ to $25 \mathrm{~cm}^{-1} / \mathrm{ms}$ [12,19]. Tsai and Wysocki reported on a modified Littrow-type EC-QCL incorporating a piezo-driven fast tilt mirror [20]. This mirror was inserted into the optical beam path between the collimating lens, collecting the emission from the anti-reflection coated QCL chip facet and the diffraction grating, the latter providing wavelength selective optical feedback. This way scan rates of up to $5 \mathrm{kHz}$ were achieved with a tuning range of up to $7 \mathrm{~cm}^{-1}$ [20]. Hugger et al. employed the same cavity configuration, but replaced the piezo-driven mirror by a fast scanning MOEMS mirror with large deflection angle [21]. The MOEMS mirror with a diameter of $3 \mathrm{~mm}$ was operated at its resonance frequency of $6 \mathrm{kHz}(167 \mu$ s per cycle). Similar to the work by Tsai and Wysocki, wavelength tuning was achieved by tilting the MOEMS mirror and thus varying the angle of incidence of the collimated beam on the fixed grating. This way, a full wavelength scan from $1070 \mathrm{~cm}^{-1}$ to $1240 \mathrm{~cm}^{-1}$ was achieved within $80 \mu \mathrm{s}$, which is half of the cycle time [21,22]. The corresponding average scan rate is approx. $2 \mathrm{~cm}^{-1} / \mu \mathrm{s}$. One limitation in this experiment was the diameter of the MOEMS mirror plate of just $3 \mathrm{~mm}$. This limitation in mirror diameter presents a challenge for the design of the external cavity in order to avoid excessive losses due to clipping of the optical beam. Therefore, MOEMS scanners with a larger diameter scanner plate and the optical grating integrated onto the scanner plate are highly desirable to realize next generation MOEMS scanner based EC-QCL. The highest tuning speed for an external cavity quantum cascade laser so far was reported by Lyakh et al. [23]. They used an acousto-optic modulator (AOM) to control the emission wavelength of a long-wave EC-QCL tunable from $\sim 8.5 \mu \mathrm{m}$ to $\sim 9.8 \mu \mathrm{m}$ with a switching time between any two wavelengths within that range of less than $1 \mu \mathrm{s}$.

In this paper, we report on recent advances in fast scanning EC-QCL employing a custom designed and fabricated MOEMS scanning grating [1,14,24]. This includes also first results on the actual use of rapid-scan EC-QCL in spectroscopic sensing experiments. Furthermore, we report recent advances in the application of EC-QCL for spectroscopic analysis and control of chemical processes [14], as well as for imaging stand-off detection of explosives [1,15-18]. 


\section{EC-QCL with MOEMS Scanning Grating}

\subsection{Setup of the MOEMS EC-QCL}

The basic setup employed in this report comprises a broadband QCL chip and a MOEMS based diffraction grating in Littrow configuration as the wavelength-selective element in the external resonator. A sketch of this MOEMS EC-QCL can be seen in Figure 1. The QCL chip is flanked by two collimation lenses $(5 \mathrm{~mm}$ diameter, numerical aperture $\mathrm{NA}=0.85)$. Internal chip modes are suppressed by an antireflection coating on the laser facet facing the MOEMS grating. An optically neutral SiN passivation layer on the opposite, out-coupling facet gives a Fresnel reflectivity of $\mathrm{R} \approx 0.28$ resulting from the modal refractive index of the QCL waveguide.

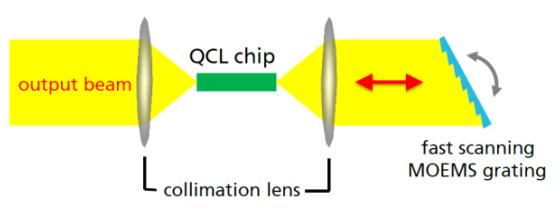

(a)

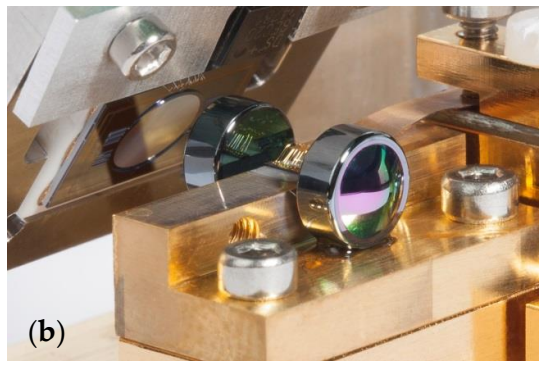

Figure 1. (a) Sketch of the EC-QCL setup with a MOEMS scanning grating in Littrow-configuration; (b) Photograph of the MOEMS EC-QCL source.

The core of the MOEMS scanning grating is a circular scanner plate with a diameter of $5 \mathrm{~mm}$, resonantly driven by an electrostatic force. Into that scanner plate a trapezoidal diffraction grating was etched, as shown in Figure 2. The MOEMS scanner was designed for a resonance frequency of $1 \mathrm{kHz}$ and maximum mechanical deflection amplitudes of $10^{\circ}$. This ensured both high spectral tuning speed and large wavelength coverage of the EC-QCL system. Torsional springs with an integrated position sensing unit provide the suspension of the scanner plate. This sensing unit consists of four piezo sensors connected to a Wheatstone bridge to achieve maximum sensitivity. This allows for continuous monitoring of the MOEMS scanner trajectory in real-time and an unambiguous determination of the wavelength of the EC-QCL while scanning, based on the phase and amplitude of the scanning grating. Due to the non-linear behavior of the torsional spring and driving force, the trajectory of the MOEMS scanner device is not strictly sinusoidal. Using a laser vibrometer setup a deviation of less than $0.35 \mathrm{~m}^{\circ}$ at scan amplitudes of $3.5^{\circ}$ was determined. A customized, USB controlled driving and control electronics board is used to operate the MOEMS scanning grating. This board provides the driving voltage for actuating the scanner plate as well as a trigger signal for each oscillation of the scanner plate which is used to synchronize the MOEMS scanner and the pulsed current driver of the QCL chip. More details on the design and fabrication of the MOEMS module can be found in reference [24].

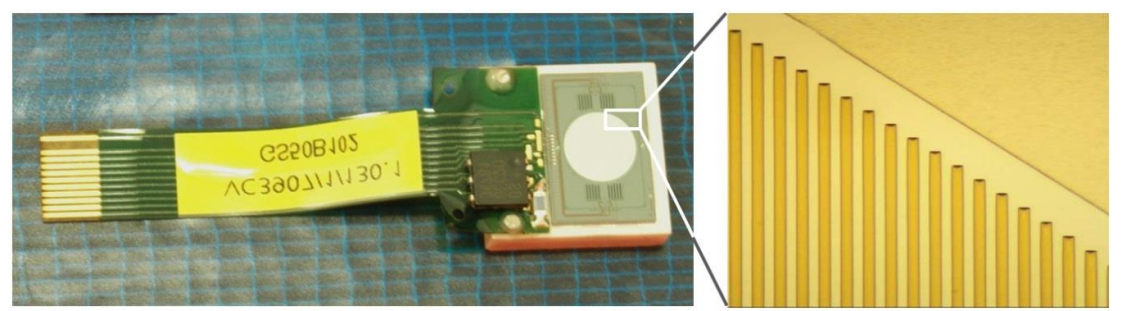

Figure 2. Photograph of the MOEMS grating module used as wavelength selective element in the EC-QCL setup (left side). The right side shows details of the V-shaped grating structure etched into the circular scanner plate. 


\subsection{Determination of the Spectral Linewidth and Wavelength Reproducibility}

Two of the most crucial aspects of any sensing system used for spectroscopy are wavelength reproducibility and spectral linewidth of the light source [25]. A spectral resolution of a few wavenumbers might be sufficient for the analysis of solids and liquids, but for gas sensing spectral linewidths well below one wavenumber are required. A planar diffraction grating as used here has a spectral resolving power directly proportional to the number of illuminated grooves on the surface of the grating [26]. Therefore, the groove density of the diffraction grating etched into the scanner plate was optimized for the wavelength tuning range of the QCL chip in order to maximize spectral resolution. For the present work, the optimization of the grating structure was performed for spectral regimes ranging from $4.4 \mu \mathrm{m}$ to $5.1 \mu \mathrm{m}$ and from $7.5 \mu \mathrm{m}$ to $10 \mu \mathrm{m}$ resulting in groove densities of $190 \mathrm{~L} / \mathrm{mm}$ and $133 \mathrm{~L} / \mathrm{mm}$, respectively. Both gratings have diffraction efficiencies well-above $90 \%$ [24].

For the experimental determination of the spectral linewidth of the MOEMS EC-QCL (for a groove density of $190 \mathrm{~L} / \mathrm{mm}$ ) we used a QCL gain chip based on a slightly diagonal active region design emitting from $2070 \mathrm{~cm}^{-1}$ to $2280 \mathrm{~cm}^{-1}$ [27]. The fast scanning EC-QCL system was operated in dynamic mode for the measurements, i.e., with the MOEMS grating resonantly driven at $975 \mathrm{~Hz}$. The QCL chip with a cavity length of $3.5 \mathrm{~mm}$ was driven in pulsed current operation (100 ns pulse length, $975 \mathrm{~Hz}$ repetition rate), synchronized to the scan frequency of the MOEMS scanner. The current pulse was triggered by a TTL signal provided by the MOEMS scanner. This way, the MOEMS EC-QCL was operated in quasi-static mode at a constant emission wavelength, tunable by the time delay between trigger signal and laser pulse. The lasing spectra were recorded using a rapid-scan FTIR spectrometer equipped with a fast photovoltaic MIR detector from VIGO and combined with a gated integrator (boxcar averager). The spectral resolution of the FTIR was set to $0.2 \mathrm{~cm}^{-1}$, the boxcar averager to a gate window of $100 \mathrm{~ns}$ and a scanning frequency of $975 \mathrm{~Hz}$ synchronized to the MOEMS scanning grating. The integration time was about $80 \mathrm{~s}$ resulting in an averaging of more than $10^{4}$ laser pulses for one single measurement.

In the upper part of Figure 3a six lasing spectra of the fast scanning MOEMS EC-QCL system can be seen (in quasi-static operation as described above), spread over the entire tuning range of the QCL gain chip. Further spectra at different wavelengths are omitted here for clarity of presentation. The expanded view in Figure 3b gives a close-up of one spectrum, for which the linewidth of the MOEMS EC-QCL can be determined to be $1.9 \mathrm{~cm}^{-1}$ at the $1 / \mathrm{e}^{2}$-cut $(13.5 \%)$, or a corresponding FWHM of about $1.1 \mathrm{~cm}^{-1}$. It has to be noted that internal longitudinal modes of the QCL chip $\left(\approx 0.4 \mathrm{~cm}^{-1}\right.$ spacing for a chip length of $3.5 \mathrm{~mm}$ ) are not separated in this measurement due to thermal chirp induced by intra pulse heating in pulsed operation [28] and the limited spectral resolution of the FTIR $\left(0.2 \mathrm{~cm}^{-1}\right)$. From the measurements presented in Figure 3 it is apparent that the linewidth, and thus the spectral resolution provided by the MOEMS EC-QCL remains constant at a value of $1.9 \mathrm{~cm}^{-1}$ over the whole spectral tuning range of the QCL chip.

To measure the wavelength reproducibility of the MOEMS EC-QCL, lasing spectra were recorded also in a true static mode, i.e., with an unplugged scanning grating in its neutral position. The MOEMS grating chip mounted on a commercial mirror holder was set manually to the respective Littrow angle for wavelength selection. The measurements were performed with the same setup described above. The trigger signal necessary to synchronize laser and boxcar averager was provided by a second resonantly driven MOEMS grating which was not placed in the external cavity. Figure $3 b$ shows that within the error margins a comparable linewidth of about $2 \mathrm{~cm}^{-1}\left(1 / \mathrm{e}^{2} \mathrm{cut}\right)$ is determined in true static operation of the MOEMS EC-QCL as in dynamic operation. Since a potential jitter of the wavelength in dynamic operation would lead to a spectral broadening when averaging over several laser pulses, we conclude that the spectral reproducibility of the laser source is within the resolution of the FTIR spectrometer $\left(0.2 \mathrm{~cm}^{-1}\right)$. 


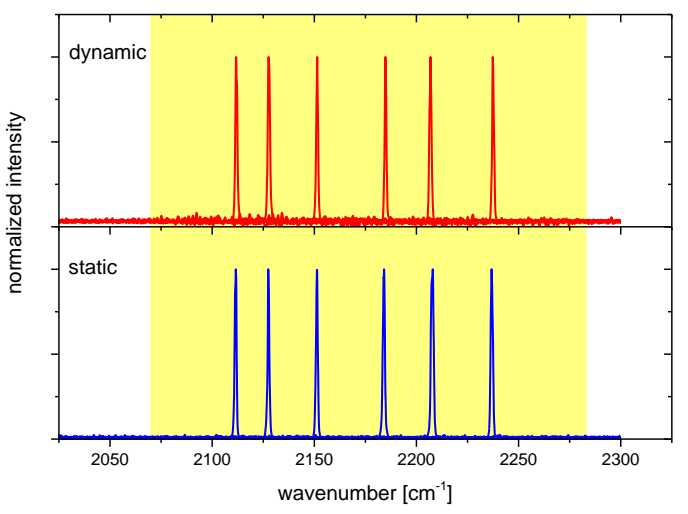

(a)

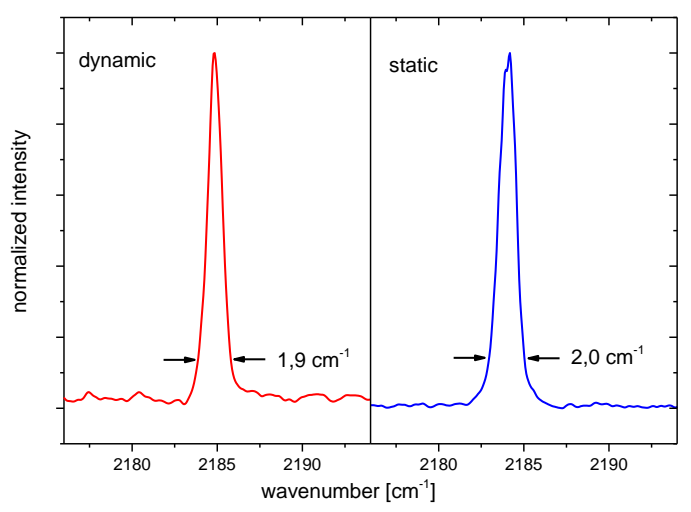

(b)

Figure 3. (a) Measurement of the spectral linewidth of the MOEMS EC-QCL at different wavenumbers. The spectral tuning range of the QCL chip is shaded in yellow. Upper graph shows the measurements in dynamic operation (quasi-static mode), i.e., with scanning grating at $975 \mathrm{~Hz}$. The lower graph shows measurements performed with a stationary grating in its neutral position, with the wavelength set manually by tilting the whole MOEMS chip; (b) Detailed view of the spectral linewidth measurements shown in Figure 3. Left side shows the measurement in dynamic operation, right side shows measurement with a static grating.

\subsection{Realtime Absorption Measurements Using MOEMS EC-QCL}

The MOEMS EC-QCL with a linewidth of $1.9 \mathrm{~cm}^{-1}$ as shown above provides sufficient spectral resolution for spectroscopy of solids and liquids. An absorption measurement on polystyrene serves as a first demonstration case to prove the capabilities of the MOEMS EC-QCL as a spectroscopic light source. Polystyrene sheets are commonly used as calibration test samples for FTIR spectrometers. A QCL chip with a heterogeneous-cascading active region design [10] tunable from $1000 \mathrm{~cm}^{-1}$ to $1300 \mathrm{~cm}^{-1}$ was used as gain material for the EC-QCL. The chip was driven in pulsed operation at $2 \%$ duty cycle (100 ns pulse length, $200 \mathrm{kHz}$ repetition rate), providing about $12 \mathrm{~mW}$ of average output power at the center of the tuning range $\left(1150 \mathrm{~cm}^{-1}\right)$. The MOEMS grating with a groove density of $133 \mathrm{~L} / \mathrm{mm}$ was oscillating at a resonance frequency of $975 \mathrm{~Hz}$ with maximum scan amplitudes of $7.5^{\circ}$. MOEMS grating and laser were driven asynchronously, i.e., the laser pulses were not synchronized to the trigger signal provided by the scanner. Again, a VIGO detector was used, which measured the intensity transmitted through the polystyrene sheet. Its signal was sent to a fast oscilloscope triggered to the TTL signal from the MOEMS grating. A total of 1024 spectra were recorded and averaged, resulting in an effective recording time of about $1 \mathrm{~s}$. The conversion of the measured data from time scale, as recorded by the oscilloscope, to wavelength scale was done post-measurement using the grating equation for an external cavity in Littrow configuration [26] and assuming a sinusoidal time-dependent grating angle given by scan amplitude and frequency of the MOEMS grating.

The red curve in Figure 4a represents the transmission spectrum of polystyrene recorded this way using the MOEMS EC-QCL. For comparison, an FTIR transmission measurement of the same polystyrene sheet is added in blue (measurement time $\sim 3 \mathrm{~min}$ ). It is apparent that the MOEMS EC-QCL can resolve the four characteristic absorption features of polystyrene in this wavelength range very well, and that its linewidth is easily sufficient for this measurement. Differences between the two spectra occur mainly at the borders of the QCL gain spectrum due to the low laser intensity at those wavelengths. Figure $4 \mathrm{~b}$ depicts the trajectory of the MOEMS scanner in red, while the tuning range of the laser chip is shaded yellow. As can be seen, the scan amplitude of $7.5^{\circ}$ of the MOEMS grating was more than sufficient to cover the full gain spectrum of the QCL chip. A unidirectional scan of the whole tuning range of the chip from $1000 \mathrm{~cm}^{-1}$ to $1300 \mathrm{~cm}^{-1}$ takes about $300 \mu \mathrm{s}$, corresponding to an actual scan rate of the MOEMS EC-QCL of $1 \mathrm{~cm}^{-1} / \mu \mathrm{s}$. Furthermore, the tuning range of the QCL chip is not symmetrically centered around the neutral position of the grating (deflection angle $0^{\circ}$ ). 
This deviation is attributed to slight misalignment of the tilt angle of the whole MOEMS chip, which determines the lasing wavelength when the grating is not deflected.

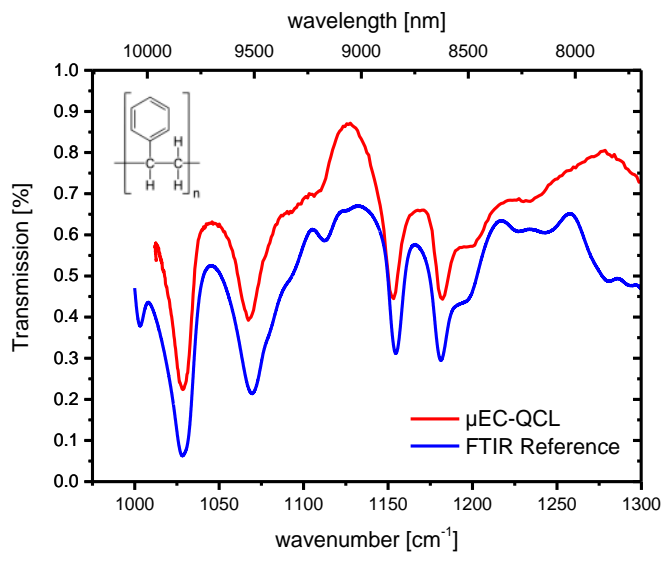

(a)

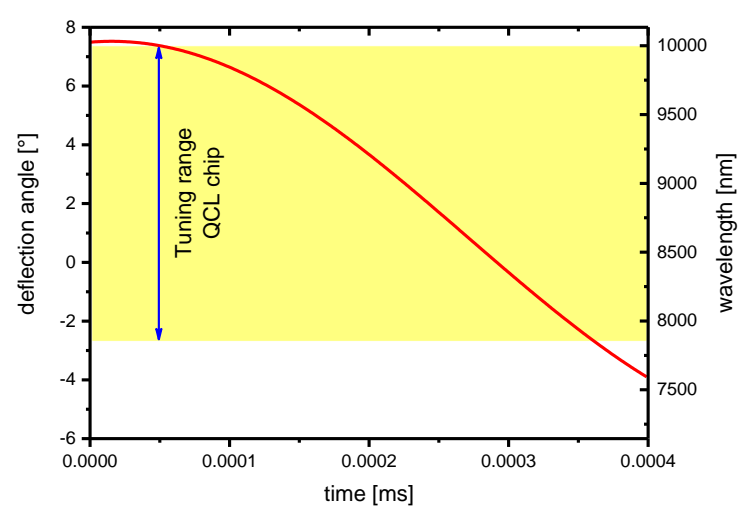

(b)

Figure 4. (a) Transmission spectrum of a polystyrene sheet recorded using the MOEMS EC-QCL as spectroscopic 1ight source at a scan frequency of $975 \mathrm{~Hz}$ (red curve). Total recording time was $1 \mathrm{~s}$ while averaging over 1024 spectra; (b) Deflection angle of the MOEMS scanner (left scale) over time, and the corresponding lasing wavelength (right scale). The tuning range of the QCL chip is shaded in yellow.

To further evaluate the potential of the MOEMS EC-QCL for spectroscopic sensing and analysis, reflectance spectra from different substances in solid phase were recorded. Shown below (Figure 5) are three reflectance spectra, taken from the explosive PETN and paracetamol as a harmless reference material, which were recorded using both a conventional EC-QCL and a MOEMS EC-QCL. The total measurement times were $20 \mathrm{~s}$ and $50 \mathrm{~ms}$, respectively, highlighting the speedup in measurement time brought about by the MOEMS EC-QCL technology without sacrificing spectral resolution and sensitivity (i.e., signal-to-noise-ratio).

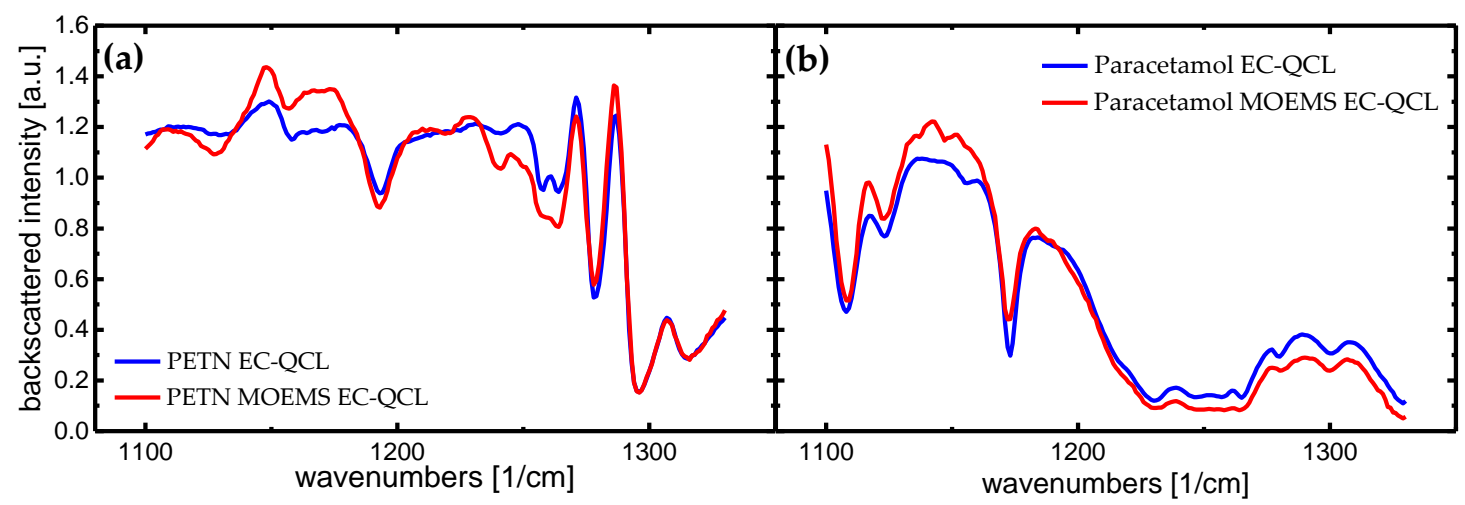

Figure 5. Reflectance spectra of different substances in solid phase - the explosive PETN (a) as well as the medical drug paracetamol (b). Spectra recorded using a conventional EC-QCL (in blue) are compared with those taken using the MOEMS-based EC-QCL (red curves, average of 100 individual spectra).

The combination of a high scan rate $\left(1 \mathrm{~cm}^{-1} / \mu \mathrm{s}\right)$ with a large tuning range $\left(>300 \mathrm{~cm}^{-1}\right)$ and a spectral resolution of $1.9 \mathrm{~cm}^{-1}$ make the present MOEMS EC-QCL technology ideally suited for real-time analysis of liquids and solids with a sub-millisecond temporal resolution. Potential application fields include on- and in-line process monitoring, analysis of kinetics in chemical reactions and real time MIR backscattering spectroscopy. 


\section{Case Studies for the Use of EC-QCL in Spectroscopic Sensing}

The following section reports on three test cases for the spectroscopic application of broadband tunable EC-QCLs in real-world scenarios. The first one is inline-monitoring of a prototypical catalytic chemical reaction, the second one is imaging MIR laser backscattering spectroscopy for the stand-off detection of residues of explosives or their precursor materials, and the third one the adoption of MIR laser backscattering spectroscopy for the contactless assessment of food quality. In all three case studies, a conventional EC-QCL module was used [18]. These modules still employ a classical optical grating mounted on a computer-controlled rotary stage as the wavelength-selective optical feedback-element [18], resulting in a maximum scanning speed in the range of $400 \mathrm{~cm}^{-1} / \mathrm{s}$.

\subsection{Inline-Monitoring of Chemical Reactions}

This case study deals with the inline analysis of an exemplary catalytic chemical reaction, the so-called Knoevenagel condensation $[14,29,30]$. This is an organic reaction that converts an aldehyde or ketone and an activated methylene to a substituted olefin with a weakly basic amine as catalyst $[29,30]$. The experimental setup used for the inline-spectroscopic analysis of this reaction consists of an EC-QCL as wavelength-tunable light source and signal and reference detectors for the acquisition of normalized transmission spectra of the analyte. Time-dependent MIR fingerprint spectra $\left(1140-1300 \mathrm{~cm}^{-1}\right)$ recorded this way are shown in Figure 6a, taken while the Knoevenagel condensation reaction was progressing. The spectra show that, despite a very low concentration of the involved reactants of $40 \mathrm{mM}$, very good signal intensity and low noise were achieved, both mostly resulting from the high energy density of the laser source. During the reaction time of about $20 \mathrm{~min}$, a clear change in the absorption spectra could be observed. A decreasing absorption band at $1160 \mathrm{~cm}^{-1}$ attributed to the aldehyde group of the educt (4-methoxybenzaldehyde) and a rising absorption band at about $1180 \mathrm{~cm}^{-1}$ attributed to the emerging $\mathrm{C}=\mathrm{C}$ double bond of the product is observed. This allows for the application of chemometric quantification procedures and determination of the product composition during the whole time of the reaction. Based on these data, the kinetics of the chemical reaction can be characterized and optimized for yield, conversion efficiency, and also the influence of the involved catalyst (not shown). This is an example of a slowly progressing reaction, i.e., over a time span of around $20 \mathrm{~min}$. This way the rate at which spectra could be recorded when using a conventional EC-QCL module was sufficient to follow the reaction dynamics. However, the availability of fast scanning MOEMS EC-QCLs (see above Section 2) allows monitoring and controlling chemical reactions on a sub-second time scale.

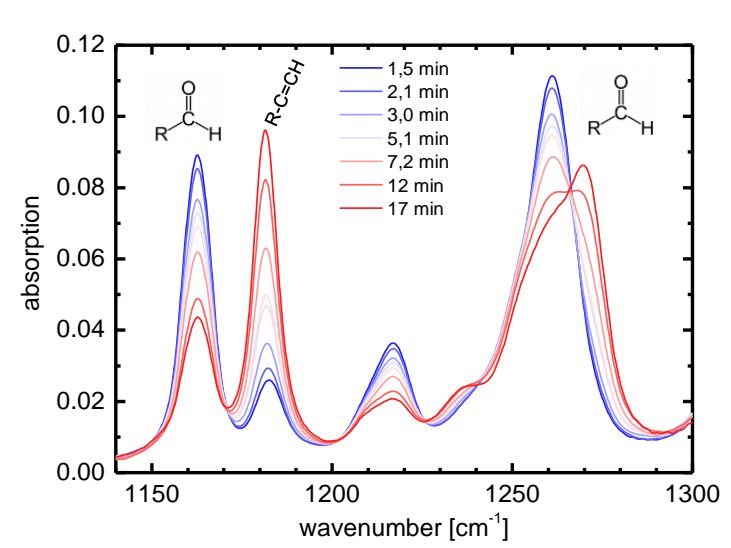

(a)

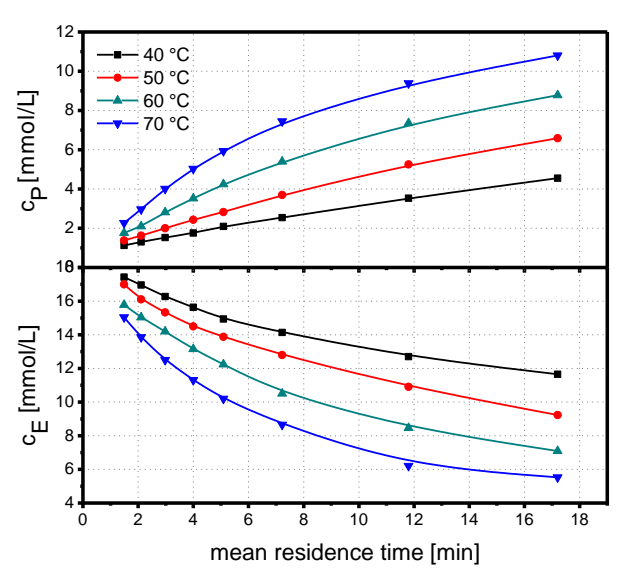

(b)

Figure 6. (a) MIR spectroscopic monitoring of a Knoevenagel condensation at $60^{\circ} \mathrm{C}$ as an exemplary catalytic chemical reaction; (b) Measurements of product and educt concentrations $\left(C_{P}, C_{E}\right)$ over time, at varying temperatures. 


\subsection{Imaging MIR Laser Backscattering Spectroscopy for the Stand-off Detection of Residues of Explosives}

The imaging MIR backscattering spectroscopy system used in field trials for the detection of explosives is shown in Figure 7. The system consists of an EC-QCL based laser illumination unit, a large aperture optical telescope coupled to a high-performance cryogenically cooled mercury cadmium telluride (MCT) MIR camera for collecting and detecting the diffusively backscattered MIR laser radiation, as well as a laptop-based control and data processing unit $[17,18,31]$. Furthermore, it comprises custom-made hardware for speckle reduction. Co-linear to the MIR telescope also the visible image of the scene under investigation is recorded.

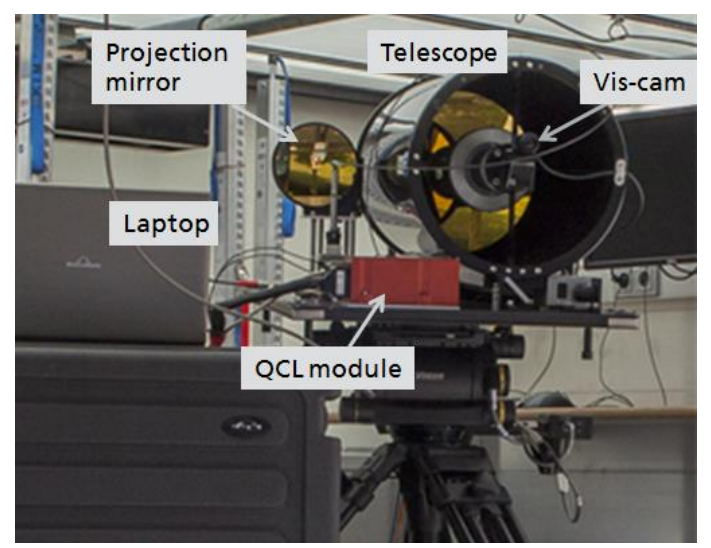

Figure 7. Stand-off detection system using a dual-chip EC-QCL module for wavelength-selective illumination and telescope mirror optics on the receiver side. The vis-cam image is overlaid over the MIR image for orientation purposes. The MCT MIR camera is located behind by the telescope.

Wavelength-tunable MIR laser illumination is performed using a compact laser module (see Figure 8a), which contains two EC-QCL units equipped with broad gain QCL chips. These QCL chips provide different, but overlapping tuning ranges. The output of the two EC-QCL units is combined via a custom made wavelength selective mirror into a single collinear output beam [2]. This way, a large overall wavelength span ranging from $7.36 \mu \mathrm{m}$ to $10.14 \mu \mathrm{m}$ (as defined by the full width at half maximum of the output power-vs.-wavelength tuning characteristic) could be covered seamlessly, corresponding to a total possible tuning range of $370 \mathrm{~cm}^{-1}$ (see Figure $8 \mathrm{~b}$ ). For the experiments below, the illumination wavelength was scanned between $7.63 \mu \mathrm{m}\left(1310 \mathrm{~cm}^{-1}\right)$ and $10 \mu \mathrm{m}\left(1000 \mathrm{~cm}^{-1}\right)$. The twin EC-QCL module was operated in short-pulse high-repetition-rate mode with an average power emitted at the maximum of the tuning curve exceeding $50 \mathrm{~mW}$ at a duty-cycle of $\sim 20 \%$.

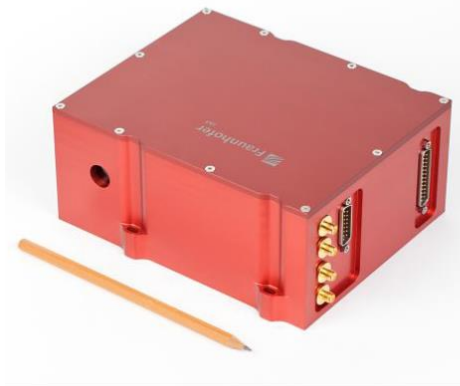

(a)

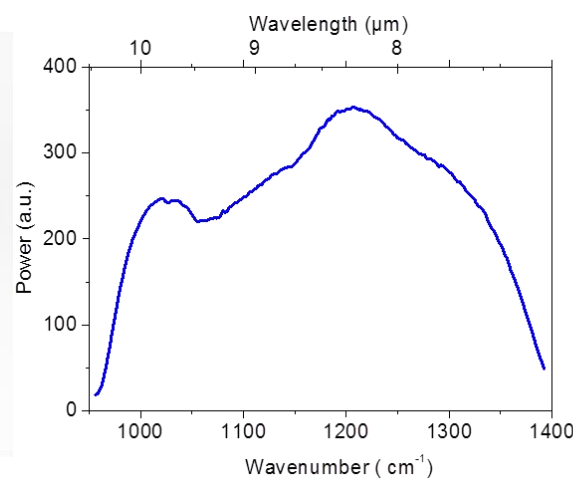

(b)

Figure 8. (a) Dual-EC-QCL module developed at our institute, containing two independent EC-QCLs combined in a single, collinear output beam; (b) Tuning range of the Dual-EC-QCL. 
Custom made data analysis software allows searching for a defined set of substances with known backscattering spectra, while a-priori-knowledge of the background material is not required [31,32]. In Figures 9 and 10 measurements of trinitrotoluol (TNT) contamination on jeans denim are shown, visualizing the ability of our detection algorithm to suppress backgrounds with arbitrary spectral fingerprints. Backscattering images illuminated by the EC-QCL at different wavelengths were recorded here from a close distance of $\sim 1 \mathrm{~m}$. Comparison to other samples with known amount of material and visual inspection suggested that the illuminated area contained a total amount of TNT on the order of $100 \mu \mathrm{g}$. Previous publications showed that over distances of a few meters, quantitates down to $10 \mu \mathrm{g}$ are detectable [33]. The backscattering spectrum of jeans denim strongly resembles that of TNT, so false alarms or interferences might be expected. However, TNT was successfully detected only in the contaminated region, and the library TNT spectrum could be recovered using linear de-mixing.

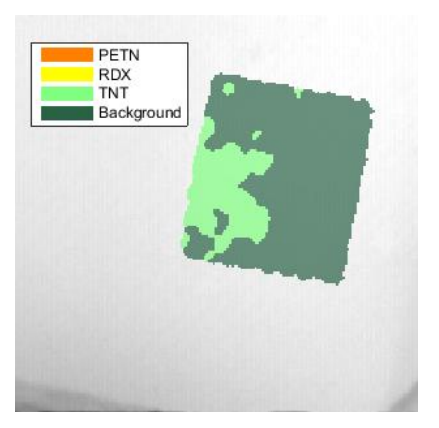

Figure 9. TNT on jeans denim detected by QCL-based MIR backscattering spectroscopy. Chemically selective image with TNT-contaminated areas shown in light green and areas where no TNT was detected in dark green.

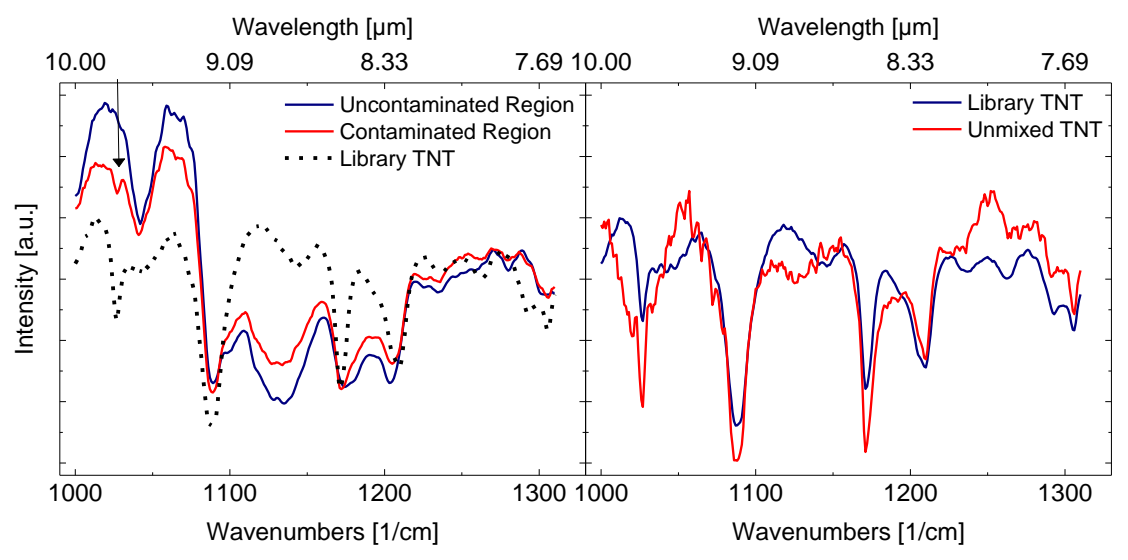

(a)

(b)

Figure 10. (a) MIR backscattering spectra taken from TNT-contaminated and not contaminated areas as well as the library backscattering spectrum of TNT. Also in the TNT-contaminated region, strong substrate contributions are found. Despite many similarities in the jeans denim and TNT spectra, the TNT library spectrum can be separated from the strong background signal and reconstructed (b).

In the following, we focus on results demonstrating stand-off detection of explosives and their precursors over larger distances of 10-20 m. These results were obtained in two series of outdoor field trials both performed as part of EU funded civilian security research projects. These trials were carried out at the test site of the Swedish Defence Research Agency (FOI). The final demonstration campaign of the EU FP 7 project EMPHASIS in September 2014 required stand-off detection ( 13 m) of traces of explosives and their precursors deliberately deposited on different spots of a car (Figure 11a). Residues of several potentially dangerous substances could be successfully identified defying adverse 
weather conditions of rain and high humidity. As an example, Figure $11 \mathrm{~b}$ shows the detection of dinitrotoluol (DNT), a precursor of TNT, on the rear tire.

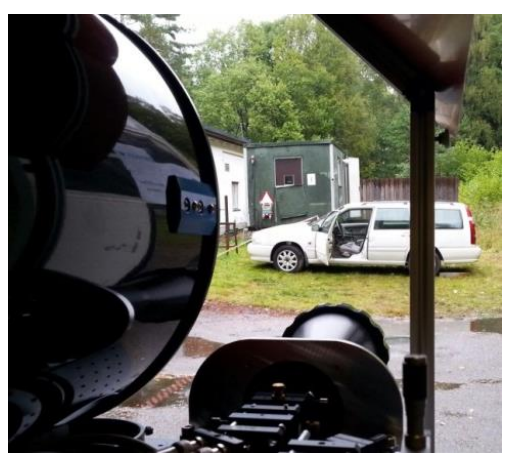

(a)

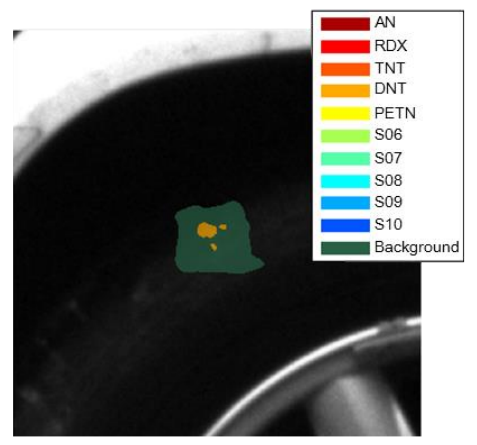

(b)

Figure 11. (a) Stand-off detection system based on MIR backscattering spectroscopy, inspecting suspect car parked at a distance of $13 \mathrm{~m}$; (b) Close-up of parts of the left rear tire of the car as detected by imaging MIR backscattering spectroscopy, showing color-coded traces of DNT, a precursor of TNT.

Another set of field trials was conducted at the FOI test site in Sweden in September 2015 within the framework of the EU FP7 project HYPERION. An improvised explosive device (IED) was detonated near a car, and we were required to identify the employed explosive by standoff detection of unexploded residues spread over the scene as "fallout" of the detonation. A crucial aspect of the scenario was the time limit of 30 min for preparation and measurement, starting from the explosion. Therefore, the detection system was mounted on a mobile platform so that measurements could be done out of a car over a distance of $17 \mathrm{~m}$. Positive detection results of ammonium nitrate (AN) contamination were obtained on different locations. Figure 12 shows the exploded vehicle and results from measurements of various areas where AN, the main constituent of the IED, was identified.

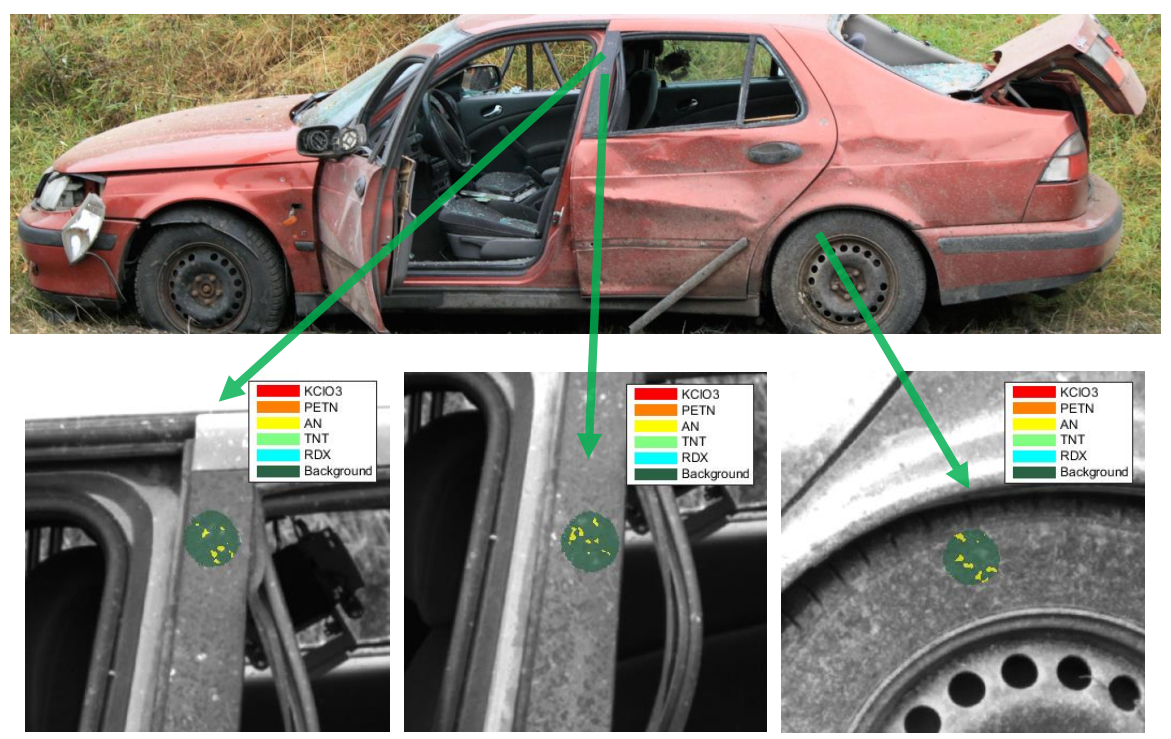

Figure 12. Photograph of a car destroyed by a nearby exploded improvised explosive device (IED), which was loaded with an AN containing explosive charge. Sections of that car where residues of unexploded ammonium nitrate were identified are expanded. AN-contaminated regions are marked in yellow; the green area indicates the illuminated area where no hazardous substances were found. Results are overlaid on the vis-image of the scene. No false alarms occurred for the other substances in the database. 
Our results were cross-checked and confirmed later by ion mobility spectrometry (IMS) analysis on samples taken by swabbing. We note that the AN contamination appeared as a thin, hardly visible layer on most parts of the car. While the actual surface concentration of $\mathrm{AN}$ is unknown, results from the analysis of swabbing samples suggest an average coverage of some $10 \mu \mathrm{g} / \mathrm{cm}^{2}$.

\subsection{Assessing Food Quality by QCL-Based MIR Backscattering Spectroscopy: Initial Results}

Even though the spectral range from $\sim 1350 \mathrm{~cm}^{-1}$ to $1800 \mathrm{~cm}^{-1}$ contains several molecular absorption bands of interest, high atmospheric absorption (mainly by water vapor) restricts standoff detection distance to less than a meter. However, this does not prove prohibitive for many applications that do not require large operation distances, e.g., for security reasons. Fast process or quality control necessitates contact-less and non-destructive identification of chemical compounds through liquids or on surfaces at close distances. The following section shows first results of food quality measurements using active MIR hyperspectral imaging. Fresh and molded peanuts are examined for their MIR backscattering characteristics and classified accordingly.

It has been reported that free fatty acid (FFA) concentration can be used as indicator for assessing food quality. High FFA concentrations in nuts and oilseed can be linked to degraded taste and microbiological contamination [34,35], e.g., by molds. The aim of the following measurements is to classify peanuts according to differences in surface concentration of FFA.

A QCL chip tunable from $1680 \mathrm{~cm}^{-1}-1800 \mathrm{~cm}^{-1}$ was used for the EC-QCL module for these measurements. For speckle reduction, the same measures were taken as for the long-range standoff detection system, but in this case a 1" germanium lens sufficed for the laser projection optics, which was about $15 \mathrm{~cm}$ from the sample. The beam spot had a diameter of about $5 \mathrm{~mm}$. The collection lens of the MCT camera ( $\mathrm{f}=100 \mathrm{~mm}, \mathrm{f} / 2)$ was $\sim 50 \mathrm{~cm}$ from the sample, and the camera had a cold filter at $4 \mu \mathrm{m}$.

FFA has characteristic absorption features in the wavelength range between $1700 \mathrm{~cm}^{-1}$ and $1800 \mathrm{~cm}^{-1}$, which can be seen in Figure 13. For these measurements, thin films of different oils were deposited on reflecting aluminium scattering plates. Thus, the reflected spectra can be interpreted as (two-way) transmission through the film. Identical but empty plates were measured from the same distance to account for spectral variations, e.g., due to atmospheric absorption. The samples were vegetable oil as a sample reference low on FFA, and FFA enriched (15\%-20\%) hazelnut-oil. As shown in Figure 12, FFA shows a pronounced absorption peak around $1710 \mathrm{~cm}^{-1}$, close by the peak associated with bound fatty acids at $1746 \mathrm{~cm}^{-1}$.

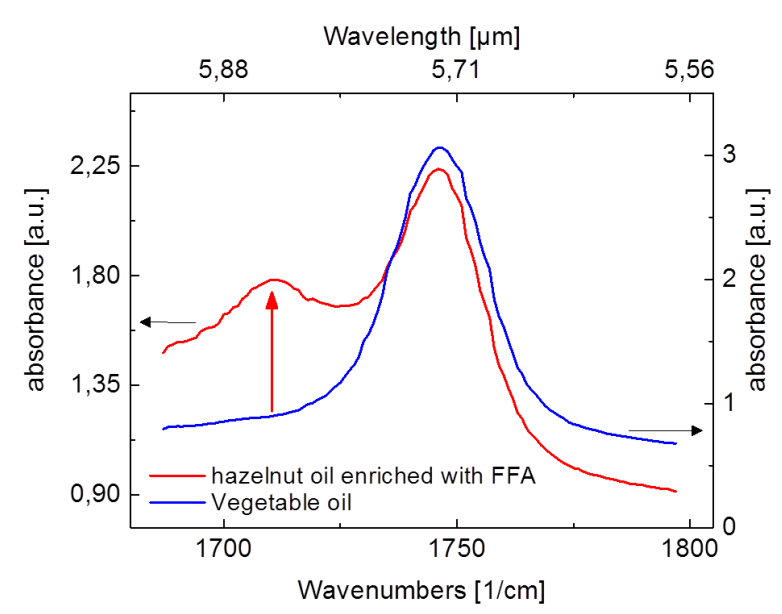

Figure 13. Absorption spectra of FFA-enriched hazelnut oil, containing both bound as well as free fatty acids (red), and vegetable oil (blue) containing mainly bound fatty acids. The feature at $\sim 1710 \mathrm{~cm}^{-1}$ is attributed to free fatty acids (FFAs). Measurements were performed with the QCL-system on thin films on aluminum substrates. 
Afterwards, this was applied to the classification of peanuts. For this, peanuts were treated by water immersion for $30 \mathrm{~s}$, followed by 5 days at $37^{\circ} \mathrm{C}, 90 \%$ relative humidity. A second set of fresh peanuts provided reference spectra. The measurements can be seen in Figure 14, showing a pronounced difference in absorption at $1710 \mathrm{~cm}^{-1}$, matching the results presented in Figure 13.

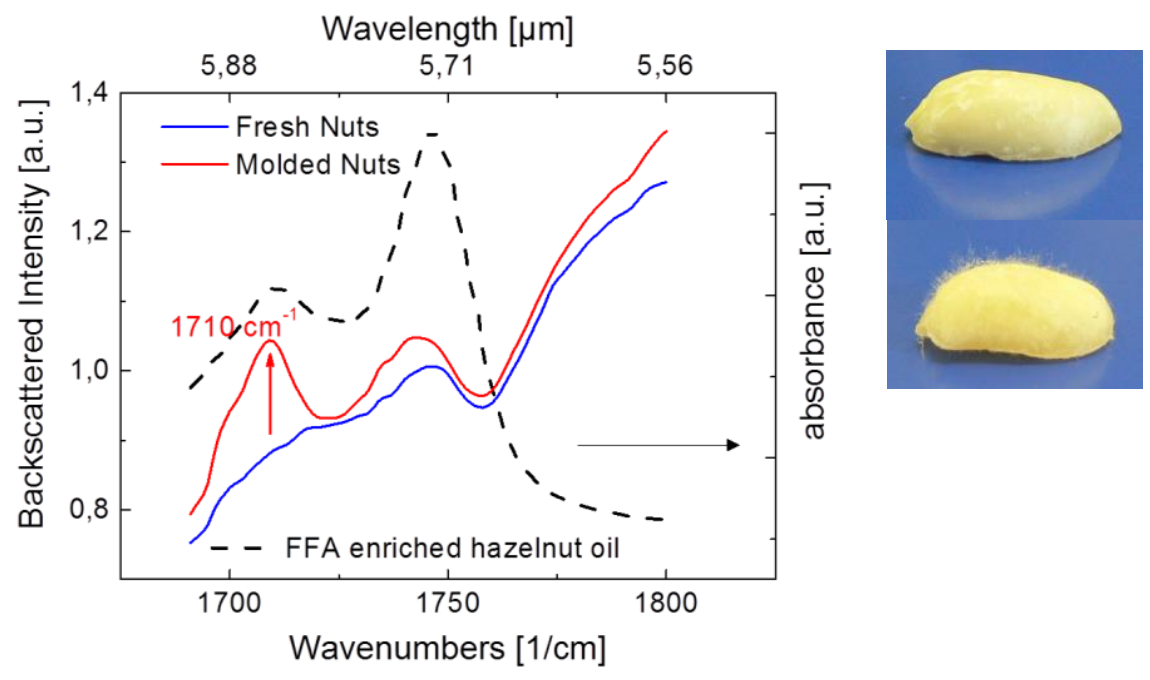

Figure 14. Solid lines: Backscattering spectra of fresh and mold-contaminated peanuts. The feature at $\sim 1710 \mathrm{~cm}^{-1}$ is only present in contaminated nuts and attributed to a higher content of free fatty acids on the nuts surface. The dotted line shows the absorbance of FFA-enriched hazelnut oil (reproduced from Figure 13) for comparison. Right: photograph of a fresh (top) and a contaminated (bottom) peanut. Any visible traces of mold, if present, were removed before the measurements were taken.

Interestingly, we find an increased intensity in the spectral region of the absorption peaks of fatty acids. Such a behavior can be understood assuming a thin surface coverage of fatty acids, if the reflectivity of the thin film increases in the vicinity of the absorption bands and the reflectivity of the underlying substrate material is low [36]. While this point has to be clarified in future work, we can conclude that the data presented in Figure 14 provide clear proof that contaminated nuts can be identified via their MIR backscattering spectrum.

\section{Conclusions}

We reported on recent advances in rapid-scan EC-QCL, equipped with a custom-made MOEMS scanning grating placed in Littrow configuration as wavelength-selective element. Operating this large aperture scanning grating ( $5 \mathrm{~mm}$ in diameter) close to its mechanical resonance frequency of $1 \mathrm{kHz}$, up to 2000 spectra per second can be obtained. Analyzing the lasing spectra of the MOEMS EC-QCL with the QCL chip driven with short-pulses and high-repetition rate both in dynamic scanning and static mode yields a laser linewidth close to $1 \mathrm{~cm}^{-1}$ FWHM in both modes of operation. This indicates that the wavelength jitter introduced by the fast scanning grating is well below the spectrometer resolution $\left(0.2 \mathrm{~cm}^{-1}\right)$. A spectral resolution of the order of $1 \mathrm{~cm}^{-1}$ is sufficient for spectroscopy on solids and liquids as well as for a range of applications in gas spectroscopy. To test the rapid-scan MOEMS EC-QCL in practical spectroscopic applications, transmission spectra were recorded from polystyrene as well as backscattering spectra from explosives and harmless reference materials. In the latter case, the data acquisition time for recording spectra with a comparable signal-to-noise ratio could be reduced from $20 \mathrm{~s}$ to $50 \mathrm{~ms}$, i.e., by a factor of 400, when switching from a conventional EC-QCL with a classical, mechanically scanned grating to the rapid-scan MOEMS EC-QCL.

In addition we reported on case studies of EC-QCL-based MIR fingerprint spectroscopy. One example was the use of EC-QCL in transmission measurements as an analytical tool for monitoring chemical reactions in real time. This particular application will benefit significantly from the use of 
rapid-scan MOEMS EC-QCL. Furthermore, we presented recent advances in EC-QCL based MIR backscattering spectroscopy (a) over larger distances of 10-20 m for the detection of hazardous materials, now tested and validated outdoors in relevant scenarios; and (b) initial tests over short distances to assess the quality of food.

Acknowledgments: This work was financed by the European FP7 projects MIRIFISens, EMPHASIS and HYPERION as well as by the Fraunhofer internal project KompLas.

Author Contributions: F. Fuchs, R. Ostendorf, M. Rattunde, S. Hugger and J. Wagner conceived and designed the experiments; L. Butschek, J. Jarvis and S. Hugger performed the experiments; J. Grahmann and A. Merten contributed the MOEMS scanners; D. Boskovic performed the measurements of the Knoevenagel condensation; K. Rieblinger and T. Tybussek were responsible for the chemical aspect of the food quality measurements; Q. Yang was responsible for laser design; C. Schilling performed laser packaging and coating; R. Ostendorf, L. Butschek, S. Hugger and J. Wagner wrote the paper.

Conflicts of Interest: The authors declare no conflict of interest.

\section{References}

1. Ostendorf, R.; Butschek, L.; Merten, A.; Grahmann, J.; Jarvis, J.; Hugger, S.; Fuchs, F.; Wagner, J. Real-time spectroscopic sensing using a widely tunable external cavity-QCL with MOEMS diffraction grating. Proc. SPIE 2016, 9755, 975507-975508.

2. Hugger, S.; Fuchs, F.; Jarvis, J.-P.; Yang, Q.; Rattunde, M.; Ostendorf, R.; Schilling, C.; Driad, R.; Bronner, W.; Aidam, R.; et al. Quantum Cascade Laser based active hyperspectral imaging for standoff detection of chemicals on surfaces. SPIE Proc. 2016, 9755, 97550A-97550A-11.

3. Faist, J. Quantum Cascade Lasers; Oxford University Press: Oxford, UK, 2013.

4. Capasso, F. High-performance midinfrared quantum cascade lasers. Opt. Eng. 2010, 49, 111102. [CrossRef]

5. Razeghi, M.; Bai, Y.; Slivken, S.; Darvish, S.R. High-performance InP-based midinfrared quantum cascade lasers at Northwestern University. Opt. Eng. 2010, 49, 111103. [CrossRef]

6. Wörle, K.; Seichter, F.; Wilk, A.; Armacost, C.; Day, T.; Godejohann, M.; Wachter, U.; Vogt, J.; Radermacher, P.; Mizaikoff, B. Breath analysis with broadly tunable quantum cascade lasers. Anal. Chem. 2013, 85, 2697-2702. [CrossRef] [PubMed]

7. Beskers, T.F.; Brandstetter, M.; Kuligowski, J.; Quintás, G.; Wilhelm, M.; Lendl, B. High performance liquid chromatography with mid-infrared detection based on a broadly tunable quantum cascade laser. Analyst 2014, 139, 2057-2064. [CrossRef] [PubMed]

8. Lambrecht, A.; Pfeifer, M.; Konz, W.; Herbst, J.; Axtmann, F. Broadband spectroscopy with external cavity quantum cascade lasers beyond conventional absorption measurements. Analyst 2014, 139, 2070-2078. [CrossRef] [PubMed]

9. Phillips, M.C.; Taubman, M.S.; Bernacki, B.E.; Cannon, B.D.; Stahl, R.D.; Schiffern, J.T.; Myers, T.L. Real-time trace gas sensing of fluorocarbons using a swept-wavelength external cavity quantum cascade laser. Analyst 2014, 139, 2047-2056. [CrossRef] [PubMed]

10. Hugi, A.; Terazzi, R.; Bonetti, Y.; Wittmann, A.; Fischer, M.; Beck, M.; Faist, J.; Gini, E. External cavity quantum cascade laser tunable from 7.6 to $11.4 \mu \mathrm{m}$. Appl. Phys. Lett. 2009, 95, 061103. [CrossRef]

11. Daylight Solutions. Available online: http://www.daylightsolutions.com/assets/005/5453.pdf (accessed on 31 March 2016).

12. Block Engineering. Available online: http://www.blockeng.com/products/lasertune.pdf (accessed on 31 March 2016).

13. Fuchs, F.; Hugger, S.; Yang, Q.; Kinzer, M.; Bronner, W.; Lösch, R.; Aidam, R.; Degreif, K.; Konz, W.; Rademacher, S.; et al. Standoff Detection of Explosives and High Sensitive Detection of Chemicals in Drinking Water with Quantum Cascade Lasers. In Lasers, Sources, and Related Photonic Devices; Optical Society of America: Miami, FL, USA, 2012; p. LM2B.6.

14. Wagner, J.; Ostendorf, R.; Grahmann, J.; Merten, A.; Hugger, S.; Jarvis, J.-P.; Fuchs, F.; Boskovic, D.; Schenk, H. Widely tunable quantum cascade lasers for spectroscopic sensing. In SPIE OPTO; Razeghi, M., Tournié, E., Brown, G.J., Eds.; International Society for Optics and Photonics: Baltimore, MD, USA, 2015; p. 937012. 
15. Hinkov, B.; Fuchs, F.; Yang, Q.K.; Kaster, J.M.; Bronner, W.; Aidam, R.; Köhler, K.; Wagner, J. Time-resolved spectral characteristics of external-cavity quantum cascade lasers and their application to stand-off detection of explosives. Appl. Phys. B Lasers Opt. 2010, 100, 253-260. [CrossRef]

16. Fuchs, F.; Hugger, S.; Kinzer, M.; Aidam, R.; Bronner, W.; Lösch, R.; Yang, Q.; Degreif, K.; Schnürer, F. Imaging standoff detection of explosives using widely tunable midinfrared quantum cascade lasers. Opt. Eng. 2010, 49, 111127. [CrossRef]

17. Fuchs, F.; Hugger, S.; Kinzer, M.; Yang, Q.; Bronner, W.; Aidam, R.; Degreif, K.; Rademacher, S.; Schnürer, F.; Schweikert, W. Standoff detection of explosives with broad band tunable external cavity quantum cascade lasers. SPIE Proc. 2012, 8268, 82681N-82681N-9.

18. Fuchs, F.; Hugger, S.; Yang, Q.; Jarvis, J.-P.; Kinzer, M.; Ostendorf, R.; Schilling, C.; Driad, R.; Bronner, W.; Bächle, A.; et al. The Wonder of Nanotechnology: Present and Future of Optoelectronics Quantum Devices and Their Applications for Environment, Health, Security and Energy; SPIE Press Book: Bellingham, WA, USA, 2013.

19. Daylight Solutions. Available online: http://www.daylightsolutions.com/assets/001/5023.pdf (accessed on 31 March 2016).

20. Tsai, T.; Wysocki, G. External-cavity quantum cascade lasers with fast wavelength scanning. Appl. Phys. B 2010, 100, 243-251. [CrossRef]

21. Hugger, S.; Fuchs, F.; Jarvis, J.; Kinzer, M.; Yang, Q.K.; Driad, R.; Aidam, R.; Wagner, J. Broadband-tunable external-cavity quantum cascade lasers for spectroscopy and stand-off detection. Proc. SPIE 2013, 8631, 645-672.

22. Yang, Q.; Fuchs, F.; Wagner, J. Quantum cascade lasers (QCL) for active hyperspectral imaging. Adv. Opt. Technol. 2014, 3, 141-150. [CrossRef]

23. Lyakh, A.; Barron-Jimenez, R.; Dunayevskiy, I.; Go, R.; Patel, C.K.N. External cavity quantum cascade lasers with ultra rapid acousto-optic tuning. Appl. Phys. Lett. 2015, 106, 141101. [CrossRef]

24. Grahmann, J.; Merten, A.; Ostendorf, R.; Fontenot, M.; Bleh, D.; Schenk, H.; Wagner, H.-J. Tunable External Cavity Quantum Cascade Lasers (EC-QCL): An application field for MOEMS based scanning gratings. Proc. SPIE 2014, 8977, 897708.

25. Alcaráz, M.R.; Schwaighofer, A.; Kristament, C.; Ramer, G.; Brandstetter, M.; Goicoechea, H.; Lendl, B. External-Cavity Quantum Cascade Laser Spectroscopy for Mid-IR Transmission Measurements of Proteins in Aqueous Solution. Anal. Chem. 2015, 87, 6980-6987. [CrossRef] [PubMed]

26. Palmer, C.; Loewen, E. Diffraction Grating Handbook; Newport Corporation: New York, NY, USA, 2005; Volume 6.

27. Yang, Q.; Lösch, R.; Bronner, W.; Hugger, S.; Fuchs, F.; Aidam, R.; Wagner, J. High-peak-power strain-compensated GaInAs/AlInAs quantum cascade lasers $(\lambda=4.6 \mu \mathrm{m})$ based on a slightly diagonal active region design. Appl. Phys. Lett. 2008, 93, 251110. [CrossRef]

28. Wysocki, G.; Lewicki, R.; Curl, R.F.; Tittel, F.K.; Diehl, L.; Capasso, F.; Troccoli, M.; Hofler, G.; Bour, D.; Corzine, S.; et al. Widely tunable mode-hop free external cavity quantum cascade lasers for high resolution spectroscopy and chemical sensing. Appl. Phys. B 2008, 92, 305-311. [CrossRef]

29. Bula, W.P.; Verboom, W.; Reinhoudt, D.N.; Gardeniers, H.J.G.E. Multichannel quench-flow microreactor chip for parallel reaction monitoring. Lab Chip 2007, 7, 1717-1722. [CrossRef] [PubMed]

30. Karabudak, E.; Mojet, B.L.; Schlautmann, S.; Mul, G.; Gardeniers, H.J.G.E. Attenuated Total Reflection-Infrared Nanofluidic Chip with $71 \mathrm{~nL}$ Detection Volume for in Situ Spectroscopic Analysis of Chemical Reaction Intermediates. Anal. Chem. 2012, 84, 3132-3137. [CrossRef] [PubMed]

31. Jarvis, J.; Fuchs, F.; Hugger, S.; Blattmann, V.; Yang, K.Q.; Ostendorf, R.; Bronner, W.; Driad, R.; Aidam, R.; Wagner, J.; et al. Trace Detection of Explosive Substances in Hyperspectral Imagery. In Proceedings of the 9th Future Security Research Conference, Fraunhofer Verlag, Berlin, 16-18 September 2014; pp. 241-247.

32. Jarvis, J.; Fuchs, F.; Hugger, S.; Blattmann, V.; Yang, K.Q.; Ostendorf, R.; Bronner, W.; Driad, R.; Aidam, R.; Wagner, J.; et al. Hyperspectral Image Analyses for Standoff Detection of Explosives. In Proceedings of the 8th Future Security Research Conference, Fraunhofer Verlag, Berlin, 17-19 September 2013; pp. 205-214.

33. Fuchs, F.; Hugger, S.; Jarvis, J.; Blattmann, V.; Kinzer, M.; Yang, Q.K.; Ostendorf, R.; Bronner, W.; Driad, R.; Aidam, R.; et al. Infrared hyperspectral standoff detection of explosives. Proc. SPIE 2013, 8710, 87100I-87100I-8.

34. Angelo, A.J.; Ory, R.L. Lipid degradation during seed deterioration. Phytopathology 1983, 73, 315-317. [CrossRef] 
35. Sztajer, H.; Maliszewska, I.; Wieczorek, J. Production of exogenous lipases by bacteria, fungi, and actinomycetes. Enzyme Microb. Technol. 1988, 10, 497. [CrossRef]

36. Hinkov, B.; Fuchs, F.; Kaster, J.M.; Yang, Q.; Bronner, W.; Aidam, R.; Köhler, K. Broad band tunable quantum cascade lasers for stand-off detection of explosives. Proc. SPIE 2009, 7484. [CrossRef]

(c) 2016 by the authors; licensee MDPI, Basel, Switzerland. This article is an open access article distributed under the terms and conditions of the Creative Commons Attribution (CC-BY) license (http:/ / creativecommons.org/licenses/by/4.0/). 